\title{
Debates críticos: os estudos de segurança e o futuro dos estudos da paz e dos conflitos*
}

\section{Critical Debates: Security Studies and the future of Peace and Conflict Studies}

André Barrinha

* Recebido em 08/08/2013. Aprovado em 15/09/2013.

1 Professor na Canterbury Christ Church University no Reino Unido e Investigador no Centro de Estudos Sociais, Universidade de Coimbra. Email: abarrinha@ces.uc.pt

\section{Resumo}

Irremediavelmente ligados pela proximidade dos respetivos objetos de estudo, Estudos de Segurança e Estudos da Paz e dos Conflitos constituíram-se e evoluíram ao longo da Guerra Fria como disciplinas antagónicas. Essa divisão operou-se sobretudo na Europa, onde Galtung e seus discípulos encaminharam o estudo das questões da guerra e da paz para uma agenda claramente normativa e crítica, enquanto o estudo das questões de segurança se centrava numa abordagem orientada para o policy-making. Como é defendido neste artigo, o final do conflito bipolar correspondeu, até certo ponto, a uma inversão de papéis, com os Estudos da Paz e dos Conflitos acomodados a um empirismo desprovido de normatividade e os Estudos de Segurança, pelo menos na Europa, a abrirem-se a novas abordagens de cariz mais crítico. É aqui sugerido que esta inversão contém, em si, importantes lições para os Estudos da Paz e da Segurança, nomeadamente sobre a centralidade da teoria na definição de uma nova agenda crítica que permita uma aproximação entre as duas disciplinas.

Palavras-Chave: Estudos da Paz e dos Conflitos. Estudos Críticos de Segurança. Teoria Crítica.

\begin{abstract}
Irredeemably connected by the proximity of their research objects, Security Studies and Peace and Conflict Studies were constituted and developed throughout the Cold War as antagonistic disciplines. This was a division mostly operated in Europe, where Galtung and his disciples directed the study of peace and war to a clearly normative and critical agenda, while the study of security remained mostly policy oriented. As argued in this article, there was, by the end of the bipolar conflict, a role inversion, with Peace and Conflict Studies accommodated to an empiricism void of any explicit normativity, whilst Security Studies, at least in Europe, opened up to new approaches of a more critical stance. It is here suggested that such inversion should provide important lessons for Peace and Conflict Studies, namely on the centrality of theory for the definition of a new critical agenda that could also contribute to bring both disciplines closer to each other.
\end{abstract}

Keywords: Peace and Conflict Studies. Critical Security Studies. Critical Theory. 


\section{Introdução}

Depois de quase duas décadas de um ensurdecedor silêncio, os Estudos da Paz e dos Conflitos (EPC) retomaram, finalmente, o debate auto-reflexivo. Artigos publicados por Patomaki (2001), Jutilla et al (2009) e Richmond (2007) reabriram um moribundo debate sobre a razão de ser da disciplina. O principal argumento deste artigo é o de que os EPC foram ultrapassados pelo arrojo intelectual dos Estudos Críticos de Segurança, vivendo um período de alguma estagnação teórica, pelo que beneficiariam em olhar de volta para estes no sentido de encontrarem respostas que levem à superação dessa mesma estagnação.

Um dos principais elogios feitos aos EPC tem sido à sua capacidade de ir além do mainstream, muitas vezes às margens profundas da academia, sem temer qualquer eventual descrédito académico. Esta busca incessante acabou por inspirar a 'viragem crítica' dos Estudos de Segurança nos anos noventa. Até à década de oitenta, os Estudos Estratégicos eram vistos como a disciplina responsável pelo estudo de questões de segurança, na altura limitadas a questões militares. Desde então, estas foram sendo progressivamente alargadas e os Estudos de Segurança assumiram um papel de maior relevo, substituindo ou sobrepondo-se aos Estudos Estratégicos (BUZAN; HANSEN, 2009; WÆVER, 2004). Em simultâneo, houve, no contexto dos Estudos de Segurança, particularmente na Europa, uma viragem crítica por parte de um conjunto de autores (KRAUSE; WILLIAMS, 1997) que acabou por constituir o que ficou conhecido como Estudos Críticos de Segurança (C.A.S.E. COLLECTIVE, 2006). No seu seio, paradigmas clássicos foram colocados em questão e novas formas de pensar a segurança desenvolvidas. Subjacente à grande maioria destas novas abordagens estava (e está) a ideia de que a segurança não é uma mera questão técnica que deve ser deixada para os especialistas para discutir, mas sim uma prática profundamente política e que deve ser entendida enquanto tal (FIERKE, 2007).

Assim, este artigo divide-se em duas partes. $\mathrm{Na}$ primeira parte será feita uma análise comparativa da evolução dos Estudos da Paz e dos Estudos de Segurança. Será aqui argumentado que ambos evoluíram alicerçados em temáticas semelhantes, tendo os Estudos da Paz evoluído no sentido de uma crítica radical ao status quo das Relações Internacionais logo no final dos anos 1960, enquanto os Estudos de Segurança só no final da década adoptaram também uma forte dimensão crítica. Na segunda parte, serão apresentados alguns argumentos no sentido do reforço da ligação entre as duas disciplinas como forma de dar um novo ímpeto crítico e teórico aos EPC.

\section{Divergências e convergências entre os Estu- dos da Paz e dos Conflitos e os Estudos de Se- gurança}

Será útil, antes de mais, debater o que são os Estudos da Paz e dos Conflitos, uma expressão que está longe de ser consensual. Para Heikki Patomaki, por exemplo, "a identidade da investigação para a paz está em discussão há 40 anos e, no entanto, nunca houve uma unanimidade clara sobre o que é nem, em bom rigor, sobre quais as suas funções" (PATOMAKI, 2001, p. 724).

No mesmo sentido, um dos pais fundadores dos EPC, Kenneth Boulding, afirmava em 1978: "julgo que podemos afirmar que o movimento de investigação para a paz produziu uma disciplina que é conhecida por diferentes nomes" (BOULDING, 1978, p. 343). Tanto Boulding (1978, p. 343) como Hugh Miall (1999, p. 12), sugerem o uso de PCS como denominador comum para a investigação em torno da conflitualidade armada. Apesar de ser uma denominação passível de diferentes críticas, trata-se de um entendimento holístico da disciplina, o que, num exercício de comparação disciplinar, tal como apresentado neste artigo, surge como lógico.

Segundo Ramsbotham, Woodhouse e Miall (2005) os EPC têm experimentado até agora quatro fases distintas. A primeira fase, ou geração, que vai de 19181945 foi essencialmente dominado pelo surgimento dos movimentos pacifistas. Esta fase coincide com as origens das Relações Internacionais como área de pesquisa: em 1919, a primeira Cátedra de Relações Internacionais (Cátedra Woodrow Wilson), foi criada na Universidade de Aberystwyth, no País de Gales. Por detrás dessa criação havia o objetivo de educar as gerações futuras para que estas olhassem para o mundo através de lentes diferentes daquelas que tinham estado na origem da Primeira Guerra Mundial. Esse idealismo do entre guerras seria manifestado noutras medidas específicas, como a criação da fracassada Sociedade das Nações.

O colapso da 'utopia da paz', juntamente com a 'viragem behaviorista' que teve lugar nas Ciências Sociais do pós-guerra, estaria na base da busca por uma legitimida- 
de científica acrescida por parte dos EPC. As Relações Internacionais, dominadas pela escola realista, tinham deixado de ser um fórum apropriado para aqueles que consideravam haver mais no entendimento da conflitualidade internacional para lá do que era oferecido pelo postulado realista. Nesse sentido, uma segunda fase na história de Estudos da Paz e dos Conflitos (1945-1965) foi marcado pelo aparecimento dos primeiros teóricos relevantes, tais como Kenneth Boulding, Johan Galtung e John Burton. Para Rogers e Ramsbotham, foi durante este período que se deu a institucionalização dos EPC (ROGERS; RAMSBOTHAM, 1999, p. 740). O estudo da conflitualidade, em grande parte restrito até então ao estudo de grandes guerras, voltou-se para a investigação científica positivista estimulados pelo Statistics of Deadly Quarrels de Quincy Wright, assim como pela re-edição do Study of War de Lewis Richardson (Cusack, 1995: 192) - os "precursores" da disciplina, tal como referido por Kenneth Boulding (1978, p. 342). O Center for Research on Conflict Resolution da Universidade do Michigan e o Center for Advanced Study in Behavioral Sciences em Stanford estavam, nessa altura, entre os primeiros programas criados com o objetivo específico de estudar a conflitualidade armada.

Na busca pela demonstração da sua relevância científica, estes académicos recorreram a outras áreas de pesquisa como a Economia, a Psicologia, a Sociologia e a Estatística a fim de produzirem investigação cientificamente 'validada'. De acordo com Terriff et al: "Foi somente através de análises 'científicas' e 'isentas' que a pesquisa para a paz [peace research] conseguiu atrair financiamento e ganhar credibilidade científica" (TERRIFF et al, 1999, p. 69). Conforme destacado por Heikki Patomaki, "a pesquisa para a paz reflectia uma crença no conhecimento científico e na capacidade de iluminar a humanidade" (PATOMAKI, 2001, p. 726).

Fora dos Estados Unidos, escandinavos e alemães adoptaram uma posição um pouco diferente. Liderados por Johan Galtung, fundador do International Peace Research Institute (Oslo, Noruega) e do Journal of Peace Research, introduziram uma abordagem estruturalista, focada nas dinâmicas de violência como determinante dos padrões de conflitualidade (SCHERRER, 1999, p. 4). Tal como os seus homólogos americanos, Galtung defendeu o desenvolvimento de pesquisas com base em análises cien-

2 Todas as citações foram traduzidas pelo autor. tíficas quantitativas, embora com objetivos diferentes. Em sua opinião, "a preocupação básica da pesquisa para a paz é a redução da violência de todos os tipos", e não apenas o estudo "científico" de conflitos (GALTUNG, 1985, p. 141). O objetivo de sua investigação era mudar o status quo, com base na ideia de que a humanidade tem uma tendência a cooperar em vez de ser inerentemente má. Nesse aspecto, Galtung estabeleceu as bases para o desenvolvimento de uma cultura da Paz; para uma pesquisa focada no potencial de cooperação e não no mero estudo da conflitualidade violenta (TERRIFF et al, 1999, p. 70).

Pode dizer-se que os Estudos de Segurança também surgiram a partir do final da Segunda Guerra Mundial como um subcampo das Relações Internacionais (WÆVER, 2004, p. 2), analisando as formas pelas quais estados foram ameaçados por outros estados através das principais teorias de Relações Internacionais. Isso aconteceu numa altura em que os Estados Unidos estavam a criar a sua estrutura de segurança (através do National Security Act de 1947) a fim de lidarem com as novas realidades securitárias do pós-guerra. Segurança Nacional passou a fazer parte do jargão diplomático e, em termos académicos, os Estudos de Segurança Nacional passaram a fazer parte dos currículos universitários nos EUA, enquanto na Europa o mesmo conteúdo passava a ser ensinado sob o rótulo de 'Estudos Estratégicos' (JONES, 1999). Como a segurança era entendida de forma restrita, principalmente como uma questão militar, os Estudos Estratégicos eram vistos como adequados para lidar com questões de segurança internacional, o que retirou o político do securitário (BUZAN, 1991, p. 345).

Até a década de sessenta, os Estudos Estratégicos e os Estudos da Paz e dos Conflitos partilharam muitos das temáticas e metodologias de investigação (DUNN, 1991): dissuasão, controlo de armas, a teoria dos jogos, entre outros. Somente a partir daí assistimos a uma crescente divergência temática e rivalidade disciplinar (BUZAN; HANSEN, 2009, p. 105), com a reorientação dos Estudos da Paz para questões como o colonialismo, as guerras de libertação e outros conflitos não convencionais: estávamos na fase de consolidação. Esta fase vai ser marcada, na Europa, pela radicalização da investigação da paz, com o conceito de "violência estrutural" (GALTUNG, 1969) a assumir um papel central na disciplina. Este conceito entendia a opressão económica como estando na base da impossibilidade de paz, levando a que a superação da es- 
trutura fosse fundamental para que essa impossibilidade assim deixasse de o ser. Opera-se assim um corte metodológico não só com os Estudos de Segurança, mas também interna aos próprios EPC, com parte destes a assumirem uma postura explicitamente normativa (PUREZA, 2011, p. 10). Tal abordagem levou a uma maior distanciamento entre os EPC europeus e a ortodoxia das Relações Internacionais; levou igualmente a uma diminuição de influência dentro da opinião pública, o que duraria até o início de 1980, quando o retorno da questão nuclear como um grande tema político na Europa, permitiu aos EPC tornarem-se "mais visíveis, mais considerados e mais relevantes" (TERRIFF et al., 1999, p. 76).

Este período de radicalização não foi seguido da mesma forma em todo o lado. De fato, como mencionado por Terriff et al. (1999, p. 75) enquanto Galtung e os seus colegas voltaram-se para a erradicação da violência estrutural, Boulding e a escola americana ainda tinham o desarmamento e o controlo de armas como seus principais focos de investigação, o que certamente deixou marcas no relacionamento transatlântico neste campo:

Tenho a forte impressão de que a última década dos estudos da paz e dos conflitos nos Estados Unidos tem sido de consolidação daquilo a que Kuhn chama de "ciência normal", sem grandes desenvolvimentos ou novas ideias, enquanto que na Europa toda a investigação para a paz tem vindo a ser desviada segundo linhas ideológicas, o que me deu a impressão de ter sido bastante infrutífero (BOULDING, 1978: 345).

$\mathrm{Na}$ década de oitenta, com o aprofundamento e alargamento do conceito, os Estudos de Segurança ganharam uma nova dinâmica dentro das Relações Internacionais. O trabalho de autores como Ullman (1983) e Buzan (1983) contribuíram para uma problematização do que é ou deve ser a segurança, remetendo o conceito para o campo do debate teórico. Os Estudos de Segurança estavam a caminho da sua 'viragem crítica'.

O que Ramsbotham et al. apelidam de fase de reconstrução (1985 a 2005) vai acabar por estabelecer os termos da investigação dos EPC no pós-Guerra Fria, vocacionando-os para a análise da multiplicidade de conflitos identitários, ou para aquilo a que Mary Kaldor (1999) apelidou de 'novas guerras'. Curiosamente, este período acabou por ser de alguma (re)convergência com os Estudos de Segurança, tal como deixado claro pelas palavras de Ken Booth:

O objecto de referência da "segurança" não devia continuar a ser, quase em exclusivo, o Estado (que enfatiza concepções militares de segurança), devendo igualmente abranger o indivíduo na base e a sociedade mundial no topo. Assim, a tradicional noção de segurança dos Estudos Estratégicos devia ser alargada, tornando-se sinónima do conceito de 'paz positiva' dos Estudos para a Paz (BOOTH, 1991, p. 341).

O surgimento dos Estudos Críticos de Segurança $^{3}$ na Europa contribuiu de forma significativa para essa mudança. Aberystwyth, Copenhaga e Paris passaram a ser nomes associados a 'escolas' de Estudos de Segurança (WÆVER, 2004, p. 6; C.A.S.E. COLLECTIVE, 2006). Neste contexto, a segurança tornou-se muito mais do que a manutenção da integridade territorial de cada estado: considerações económicas e ambientais foram, por exemplo, adicionadas à equação. No cerne destas novas abordagens estava o "reconhecimento da ideia de que a segurança é, essencialmente, um conceito derivado; o que significa, simplesmente, que teorias rivais sobre a política mundial produzem diferentes conceptualizações sobre o que é segurança na política mundial” (BOOTH, 2005, p. 13).

De certo modo, os Estudos Críticos de Segurança passaram, no início dos anos noventa, por um processo semelhante àquele a que os EPC tinham passado nos anos sessenta: concentrando-se no conceito que dá nome à disciplina em vez de se limitarem ao seu enquadramento no contexto mais geral das teorias das Relações Internacionais. Até então, "não tinha havido qualquer discussão explícita do conceito de 'segurança' em si: o que era contestado eram as explicações realistas para o comportamento dos estados na área da segurança" (BUZAN; HANSEN, 2009, p. 17).

Algumas das ideias que se tornaram dominantes no quadro dos Estudos Críticos de Segurança começaram a ser desenvolvidas no contexto de centros de investigação no campo dos Estudos da Paz e dos Conflitos, como foi o caso do Copenhagen Peace Research Institute (COPRI) (MIALL, 1999, p. 23). Também durante este período houve várias obras sobre segurança no Terceiro Mundo (AYOOB, 1995), que desafiavam os pressupostos mais clássicos sobre o conceito (THOMAS, 1991, p. 274). Na verdade, esta é uma questão que tem acompanhado

\footnotetext{
3 Conforme destacado na introdução ao primeiro livro que compila estas diferentes abordagens, o termo crítico foi introduzido para significar "mais uma orientação para a disciplina que um rótulo teórico preciso” (KRAUSE; WILLIAMS, 1997, p. 10-11).
} 
os Estudos de Segurança desde então. Apesar de mais aberta a abordagens alternativas, as obras realizadas sob a égide geral dos seus estudos críticos continuam a ser frequentemente acusadas de desenvolverem modelos e estruturas centradas na realidade europeia, colocando de lado as questões de segurança doutras partes do mundo (WALKER, 2007; WILKINSON, 2007).

Segundo Ramsbotham et al (2005, p. 33), estamos agora numa quinta fase dos EPC em que as especificidades da conflitualidade e da paz no século XXI têm de ser consideradas enquanto tal (e não enquanto a mera continuação de dinâmicas anteriores). Ainda na opinião destes autores, isso abre perspectivas para a cooperação entre ambos os campos com as suas agendas de investigação a tornam-se cada vez mais semelhantes com o foco em temas como o terrorismo e o meio ambiente (RAMSBOTHAM ET AL, 2005, p. 33). Contudo, tal como sugerido neste artigo, mais do que uma questão temática, esta aproximação devia corresponder a uma renascido interesse pela teoria e por uma atitude crítica (PUREZA, 2011) por parte dos EPC.

\section{Estudos da Paz e dos Conflitos e Estudos de Segurança: uma relação cheia de ligações}

Como acabamos de ver, os Estudos da Paz e dos Conflitos e os Estudos de Segurança têm vindo a percorrer caminhos que tanto têm sido convergentes como antagónicos. Olhando para trás, seria importante salientar três ocasiões diferentes em que essa convergência ocorreu.

Antepassados. Pode-se argumentar que ambas as disciplinas compartilham as mesmas origens: o pós-Primeira Guerra Mundial e o consequente movimento pacifista que defendeu o estudo de guerras passadas a fim de entender melhor como evitá-las no futuro e que esteve na base da criação da disciplina de Relações Internacionais. A análise dos conflitos era então limitada ao estudo das grandes guerras inter-estatais e o conceito de 'segurança' ainda não tinha substituído o conceito de 'interesse nacional' no vocabulário da política internacional.

A 'viragem científica'. O desejo de reconhecimento de uma certa legitimidade académica levou ambos os campos, no início dos anos cinquenta, a desenvolverem metodologias e abordagens baseadas nas ciências naturais, bem como a concentrarem as suas atenções na temá- tica que mais facilmente garantia financiamento externo e a atenção da opinião pública: a corrida às armas nucleares. Uma vez que o tópico de investigação era comum e as metodologias semelhantes, era por vezes difícil distinguir o trabalho feito pelos Estudos da Paz e dos Conflitos da investigação dos Estudos de Segurança (Nacional).

O alargamento da agenda de investigação. A partir do final da década de 1980, os Estudos de Segurança começaram a ampliar a sua agenda de investigação, incluindo questões até então fora do seu campo de interesses, como o ambiente, as migrações ou a saúde. Como anteriormente visto, esta mudança foi, até certo ponto, impulsionada pelos Estudos da Paz e dos Conflitos. O fato dos Estudos (Críticos) de Segurança estarem mais concentrados em seguir o rótulo 'segurança' onde quer que este fosse usado, tornou, em princípio, mais possível o estabelecimento de um mais ativo diálogo com os Estudos sobre Paz e Conflitos.

Mas que tipo de diálogo? Defende-se neste artigo, que esse diálogo passe, não pelo explorar da dimensão crítica comum às duas disciplinas, como também ao reforço dos laços teóricos, que permita, no caso dos Estudos da Paz e dos Conflitos, um renovado empenho conceptual e uma acrescida capacidade de problematização dos fenómenos da paz e da guerra no século XXI. Como diz José Manuel Pureza,

a ciência normal, nos estudos para a paz, neste início do século xxi, é uma combinação de falta de contaminação pelos grandes movimentos que animam o debate teórico em relações internacionais ou em estudos sobre a segurança e de uma auto-reflexividade tranquilizadora e triunfalista da linha dominante desta área face à cooptação do seu universo conceptual pelo projecto de paz liberal, entretanto transformado em gramática do poder em escala mundial (PUREZA, 2011, p.17).

No mesmo sentido Carlos Yordán afirma: “é surpreendente que a Análise de Conflitos ainda não tenha sido afectada pela 'viragem crítica' das RI" (YORDÁN, 2003, p. 60). Olhando para a questão levantada por um dos autores mais relevantes dentro de Paz e Conflitos Estudos, Christopher Mitchell, tal ausência pode, provavelmente, ser mais claramente compreendida:

Sou um positivista e empirista inveterado, por isso acredito (mas não o posso, em última análise, comprovar para lá de qualquer sombra de dúvida) que há um mundo 'lá fora' cheio de coisas, algumas das quais nós concordamos em chamar de 'conflitos', que valem a pena tentar 
analisar e compreender. A ideia de Foucault que de alguma forma nós 'criamos' ou 'construímos' este mundo parece-me ser fundamentalmente errado e enganador - e também uma das ideias intelectualmente mais arrogantes que eu alguma vez encontrei. Nós não 'construímos' o mundo, nem a parte dele que estamos interessados em tentar entender (MITCHELL, 2001, p. 6).

Conforme destacado pelo C.A.S.E Collective (2006, p. 462) e por Vivienne Jabri (1996, p. 55), a 'ortodoxia' dos EPC baseia-se num excessivo ênfase na análise empírica a partir da qual é possível descobrir regularidades e deduzir explicações gerais; uma abordagem que é muito 'limitada' (CASE, 2006, p. 462) e teoricamente 'pobre' (MIALL, 1999, p. 18; REIMANN, 2002, p. 391) no âmbito das ciências sociais contemporâneas. Como também destaca Jabri:

O campo tem sido tão dominado por uma orientação positivista que as questões de ontologia e epistemologia foram amplamente ignoradas, uma vez que os métodos das ciências naturais foram assumidos como aplicáveis ao estudo dos fenómenos sociais, como é o caso da conflitualidade (JABRI, 1996, p. 22).

Como mencionado no início do artigo, esta imagem já não é totalmente representativa da disciplina $\mathrm{e}$ temos hoje um renovado impulso crítico, providenciado entre outros, pelo trabalho desenvolvido no Humanitarian and Conflict Response Institute da Universidade de Manchester, por autores como Roger Mac Ginty e Oliver Richmond. No entanto, a 'normalidade' da 'ciência' ainda é hoje largamente baseada em cânones positivistas e declaradamente empiricistas.

Um aproximar dos EPC aos Estudos Críticos de Segurança poderia, nesse sentido, contribuir para o fortalecer dessa agenda crítica. Sugere-se, neste artigo, que tal pode passar por dois processos: de aprendizagem e de diálogo.

Aprendizagem. No campo crítico dos Estudos de Segurança, os seus principais autores estão em frequente contato com outras áreas do pensamento, nomeadamente a Filosofia e a Teoria Política. Por exemplo, a chamada Escola de Aberystwyth é fortemente influenciada pelos pensadores da Escola de Frankfurt. No entanto, seria abusivo entender as obras de Ken Booth ou até mesmo de Richard Wyn Jones como correspondendo a meras aplicações da Teoria Social Crítica ao estudo da segurança. Estas abordagens têm em comum "a identificação e denúncia da despolitização da sociedade e do mundo académico"
(CASE COLLECTIVE, 2006, p. 445), mas avançam em direcções distintas. No mesmo sentido, trabalhos de autores como Didier Bigo ou Michael Dillon são fortemente influenciados pelas ideias do pensador francês Michel Foucault. Mas, não só Foucault é apenas uma influência (mesmo que bastante forte no caso de Dillon) na complexa teia de pensamento desses autores, como essas ideias são usadas em diferentes perspectivas, fazendo com que o pensamento de Bigo seja significativamente diferente do de Dillon. Para além da incorporação do pensamento de autores exteriores aos Estudos de Segurança, estes autores da chamada 'viragem crítica' têm igualmente uma forte preocupação em debater entre si os principais conceitos e teorias da disciplina. Um bom exemplo disso é a ampla produção bibliográfica da última década sobre a Escola de Copenhaga, tanto por seguidores, como por críticos (BALZACQ, 2011).

Quando se trata dos EPC, Rogers e Ramsbotham (1999, p. 752) salientam o quão 'escasso' tem sido esse diálogo intradisciplinar. A reprodução destas práticas de produção e debate teórico dos Estudos Críticos de Segurança por parte dos EPC contribuiria, certamente, para uma reflexividade acrescida da disciplina, assim como para o seu enriquecimento teórico. Esta aprendizagem não deve ser acrítica e há certamente passos na evolução dos Estudos Críticos de Segurança que os EPC poderiam evitar. Mas isso é algo só possível a partir do momento em que se aceita a necessidade do processo.

Diálogo. Para além de aprender, os EPC também teriam algo a ensinar, o que só pode acontecer a partir de uma interacção directa entre as duas disciplinas. É a esse respeito extraordinário como apesar da aproximação temática entre as duas áreas, estas se mantenham institucionalmente tão separadas. Revistas científicas, ensino e associações de profissionais permanecem claramente divididas; os autores chave duma área são em larga medida desconhecidos da outra e são pontuais e assinalados enquanto tal (como este artigo) os esforços de diálogo direto sobre o que se faz num e noutro lado. A esse respeito, tão importante como a interacção entre os EPC e os Estudos Críticos de Segurança seria eventualmente perceber as razões por detrás da ausência dessa mesma interacção; o que isso diz das disciplinas e das suas formas concretas de produção de conhecimento. Esse esforço contribuiria, por fim, para alguma recentragem dos Estudos Críticos de Segurança nas problemáticas da conflitualidade arma- 
das (NEUMANN, 1998), assim como obrigaria os EPC a aceitar o envolvimento em debates teóricos que necessariamente colocariam em causa e mostrariam a contingência conceptual dos modelos vigentes na disciplina.

\section{Conclusão}

Partindo de uma preocupação já igualmente demonstrada por outros autores (JUTILLA et al, 2009; PATOMAKI, 2000; PUREZA, 2011; RICHMOND, 2007), este artigo pretendeu mostrar como os EPC perderam algum do seu fulgor crítico, que esteve justamente na base do desenvolvimento de outras abordagens críticas dentro e fora das Relações Internacionais. Foi aqui deixado claro que os EPC e os Estudos de Segurança têm uma história em comum que importa conhecer e perceber no sentido da evolução intelectual das duas disciplinas, mas, sobretudo, no sentido da intensificação do pendor teórico e crítico dos estudos relativos às questões da paz e dos conflitos. É para isso importante que os EPC tenham a humildade disciplinar e a reflexidade necessária para perceberem em que momento exacto da sua evolução se perderam esses seus dois alicerces chave da sua construção - a teoria e a crítica - e como uma maior interacção e aprendizagem com os Estudos Críticos de Segurança pode ajudar nesse mesmo trabalho de resgate. Como sugerem Buzan e Hansen (2009), para os EPC a alternativa a essa opção pode bem ser a sua possível irrelevância disciplinar.

\section{Referências}

AYOOB, Mohammed. The Third World Security Predicament. Boulder: Rienner, 1995.

BALZACQ, Thierry(Ed.). Securitization Theory: how security problems emerge and dissolve. New York: Routledge, 2011.

BOOTH, Ken. War, Security and Strategy: towards a doctrine for stable peace. In: BOOTH, Ken (Ed.). New Thinking About Strategy and International Relations. London: Harper Collins, 1991. p. 335-376.

BOOTH, Ken. Critical Explorations. In: BOOTH, Ken (Ed.). Critical Security Studies and World Politics. London: Lynne Rienner Publishers, 2005. p. 1-18

BOULDING, Kenneth. Future Directions in Conflict and Peace Studies. The Journal of Conflict Resolution. Stanford, California, USA, v. 22, n. 2, p. 342-354,1978.
BUZAN, Barry. People, States and Fear. The National Security Problem in International Relations. Brighton: Wheatsheaf Books, 1983.

BUZAN, Barry. Is international security possible? In: BOOTH, Ken (Ed.) New Thinking About Strategy and International Relations, London: Harper Collins, 1991.

BUZAN, Barry; HANSEN, Lene Hansen. The Evolution of International Security Studies. Cambridge: Cambridge University Press, 2009.

BUZAN, Barry; WÆVER, Ole. WILDE, Jaap de. Security: a new framework for analysis. Boulder, Colorado, USA: Lynne Rienner, 1998.

C.A.S.E. COLLECTIVE. Critical Approaches to Security in Europe: A Networked Manifesto. Security Dialogue, Oslo, Norway, v. 37, n. 4, p. 443-487, 2006.

CUSACK, Thomas. On the Theoretical Deficit in the Study of War In: BREMER, Stuart; CUSACK, Thomas (Ed.). The Process of War. Advancing the Scientific Study of War. Amsterdam: Gordon and Breach Publishers, 1995. p. 191-202.

DUNN, David. Peace Research versus strategic studies. In: BOOTH, Ken (Ed.). New Thinking About Strategy and International Relations. London: Harper Collins, 1991. p. 56-72.

FIERKE, Karin. Critical Approaches to International Security. Cambridge: Polity Press, 2007.

GALTUNG, Johan. Twenty-Five Years of Peace Research: ten challenges and some responses. Journal of Peace Research, Oslo, Norway, v. 22, n. 2, p. 141-158, 1985.

JABRI, Vivienne. Discourses on Violence. Conflict analysis reconsidered. Manchester: Manchester University Press, 1996.

JONES, Richard. Security, Strategy and Critical Theory. London: Lynne Rienner Publishers, 1999.

JUTILA, Matti; PEHKONEN, Samu; VAYRYNEN, Tarja Vayrynen. Resuscitating a discipline: an agenda for critical peace research. Journal of Peace Research, Oslo, Norway, v. 36, n. 3, p. 623-640, 2008.

KRAUSE, Keith; WILLIAMS, Michael. Critical Security Studies. Concepts and Cases. London: Routledge, 1997.

MIALL, Hugh. What do peace studies contribute distinctively to the study of peace. Political Studies Association Conference, Nottingham, United Kingdom, march, 1999. 
MITCHELL, Christopher. Conflict Analysis, Conflict Resolution and Politics. In: FISCHER, Martina; SCHMELZLE, Beatrix. Berghof Handbook for Conflict Transformation. 2006. Disponível em: <www.berghofhandbook.net>. Acesso em: 12 mar. 2013

NEUMANN, Iver. Identity and the Outbreak of War: or why the Copenhagen School of Security: studies should include the idea of 'violisation' in its framework of analysis. The International Journal of Peace Studies. Oslo, Norway, v. 3, n.1, 1998.

PATOMAKI, Heikki . The Challenge of Critical Theories: Peace Research at the Start of the New Century. Journal of Peace Research. Oslo, Norway, v. 38, n. 6, p.723-737, 2001.

PUREZA, José Manuel. O desafio crítico dos Estudos para a Paz. Relações Internacionais, Lisboa, Portugal, $\mathrm{n}$. 32, p. 5-22, 2011.

RICHMOND, Oliver. Critical Research Agendas for Peace: The Missing Link in the Study of International Relations. Alternatives, O)ntario, Canada, v. 32, n., p. 247274, 2007.

ROGERS, Paul; RAMSBOTHAM, Oliver. Then and Now: Peace Research: past and Future. Political Studies, United Kingdom, n. 47, p. 740-754, 1999.

SCHERRER, Christian P. Peace Research for the 21st Century: A Call for Reorientation and New Research Priorities. Copenhagen: Copenhagen Peace Research Institute, 1999.
TERRIFF, Terry; CROFT, Stuart; JAMES, Lucy; MORGAN, Patrick. "Peace Studies". In: Security Studies Today. Cambridge, United Kingdom: Polity Press, 1999. p. 65-81.

THOMAS, Caroline. New directions in thinking about security in the Third World. In: BOOTH, Ken (Ed.). New Thinking About Strategy and International Relations. London: Harper Collins, 1991. p. 267-289.

ULLMAN, Richard. Redefining Security. International Security. Local, v. 8, n. 1, p. 129-153, 1983.

WÆVER, Ole. New Schools. In: Security Theory and their Origin between Core and Periphery", Paper presented at the annual meeting of the International Studies Association, Montreal, March 17-20, 2004.

WALKER, R. B. J. Security, Critique, Europe. Security Dialogue, Oslo, Norway, v. 38, n. 1, p. 95-103, 2007.

WILKINSON, Claire. The Copenhagen School on Tour in Kyrgyzstan: is securitization theory useable outside Europe? Security Dialogue, Oslo, Norway, v. 38, n. 1. p. 5-25, 2007.

YORDÁN, Carlos L. Towards Critical Theories of Conflict Analysis: what the Critical Turn in International relations can teach conflict analysis", Journal of International and Area Studies, Oslo, Norway, v.10, n.1, p. 59-74, 2003. 